\begin{tabular}{|c|l|}
\hline Title & Charged vortices in high-temperature superconductors probed by NMR \\
\hline Author(s) & Kumagai, Ken-ichi; Nozaki, Koji; Matsuda, Y uji \\
\hline Citation & $\begin{array}{l}\text { PHY SICAL REVIEW B, 63, 144502 } \\
\text { https://doi.org/L0.1103/PhysRevB.63.144502 }\end{array}$ \\
\hline Issue Date & 2001 \\
\hline Doc URL & http://hdl.handle.net/2115/5892 \\
\hline Rights & Copyright $\odot 2001$ A merican Physical Society \\
\hline Type & article \\
\hline File Information & PRB63.pdf \\
\hline
\end{tabular}

Instructions for use 


\title{
Charged vortices in high-temperature superconductors probed by NMR
}

\author{
Ken-ichi Kumagai, ${ }^{1,3}$ Koji Nozaki, ${ }^{1}$ and Yuji Matsuda ${ }^{2,3}$ \\ ${ }^{1}$ Division of Physics, Graduate School of Science, Hokkaido University, Kita-ku, Sapporo 060-0810, Japan \\ ${ }^{2}$ Institute for Solid State Physics, University of Tokyo, Kashiwanoha, 5-1-5, Kashiwa, Chiba 277-8581, Japan \\ ${ }^{3}$ CREST, Japan Science and Technology Corporation, Kawaguchi, Saitama 332-0012, Japan
}

(Received 6 October 2000; published 16 March 2001)

\begin{abstract}
We report experimental evidence that a vortex in high-temperature superconductors (HTSC) traps a finite electric charge from the high-resolution measurements of the nuclear quadrupole frequencies. In slightly overdoped $\mathrm{YBa}_{2} \mathrm{Cu}_{3} \mathrm{O}_{7}$ the vortex is negatively charged by trapping electrons, while in underdoped $\mathrm{YBa}_{2} \mathrm{Cu}_{4} \mathrm{O}_{8}$ it is positively charged by expelling electrons. The sign of the trapped charge is opposite to the sign predicted by the conventional BCS theory. Moreover, in both materials, the deviation of the magnitude of charge from the theory is also significant. These unexpected features can be attributed to the electronic structure of the vortex in HTSC.
\end{abstract}

DOI: 10.1103/PhysRevB.63.144502

PACS number(s): 74.60.Ec, 76.60.Gv

\section{INTRODUCTION}

One of the most important physical properties of the vortex created in type-II superconductors is that a vortex line can support a magnetic flux with a flux quantum $\Phi_{0}$ $=h c / 2 e\left(=2.07 \times 10^{-7} \mathrm{Oe} \mathrm{cm}^{2}\right) .{ }^{1}$ This fact has been confirmed experimentally more than 4 decades ago. On the other hand, it is only very recent that another prominent feature, namely, the possibility that a vortex of the superconductor can accumulate a finite electric charge as well, has come to be realized. ${ }^{2-5}$ The vortex charge appears as a result of the chemical potential difference between the vortex core and the region away from the vortex core. It should be emphasized that the sign and magnitude of this charge is closely related to the microscopic electronic structure of the vortex, which in turn reflects the total fundamental nature of the superfluid electrons and the low-energy excitation of the condensate. Moreover, it has also been pointed out recently that the vortex charge strongly affects the dynamical properties of the vortex. ${ }^{6-10}$ For instance, the origin of the vortex Hall anomaly has been attributed to the vortex charge. Though the clarification of the issue of the vortex charge serves as an important test of the predictions for the vortex electronic structure and the dynamics, it has never been examined experimentally so far. This is mainly because in conventional superconductors the magnitude of the accumulated charge within the core is very small and is extremely difficult to observe.

In this paper we report a straightforward attempt to identify the vortex charge in high-temperature superconductors (HTSC) by high-resolution measurements of the nuclear quadrupole frequency $\nu_{Q}$, which is very sensitive to the local charge density. We show that a vortex in HTSC indeed traps a small but finite electronic charge as well. In slightly overdoped $\mathrm{YBa}_{2} \mathrm{Cu}_{3} \mathrm{O}_{7}$ the vortex is negatively charged, while in underdoped $\mathrm{YBa}_{2} \mathrm{Cu}_{4} \mathrm{O}_{8}$ it is positively charged. The sign of the trapped charge is opposite to the sign predicted by the conventional BCS theory. Moreover, in both materials, the accumulated charge is much larger than expected in the ordinary superconductors. We discuss several possible origins for these discrepancies.

\section{VORTEX CHARGE}

We first briefly introduce the vortex charge in type-II superconductors. In the core of the conventional superconductors, the distance between each discrete energy level of the quasiparticles in the Andréev bound states is merely of the order of a few $\mathrm{mK}$. Thus it is sufficient to view the energy levels as forming a continuous spectrum, just like in a normal metallic state. ${ }^{1,11}$ Generally the chemical potential $\mu$ in the superconducting state differs from that in the normal state if an electron-hole asymmetry is present. Assuming therefore that the vortex core is a normal metallic region surrounded by the superconducting materials, this difference in $\mu$ is expected to arise and should lead to the redistribution of the electrons. In order to maintain the same electrochemical potential on both sides, the charge transfer occurs between the core and the outside.

In the framework of the BCS theory, taking into account the metallic screening effect, the charge accumulated within the vortex core $Q_{\xi}$ per layer, normal to the magnetic field is given as

$$
Q_{\xi} \approx \frac{2 e k_{F} s}{\pi^{3}}\left(\frac{\lambda_{T F}}{\xi}\right)^{2}\left(\frac{d \ln T_{c}}{d \ln \mu}\right)
$$

where $\lambda_{T F}$ is the Thomas-Fermi screening length, $s$ is the interlayer distance, $\mu$ is the chemical potential, and $e(>0)$ is the electron charge. ${ }^{3}$ The sign of the core charge is determined by $d \ln T_{c} / d \ln \mu$, which represents the electron-hole asymmetry. Outside the core, the charges with opposite sign screen the core charge similar to a charged particle in a metal. Far outside the core, these screening charges decay gradually with a power-law dependence as $r^{-4}$ [see Fig. $1(\mathrm{a})]^{3,9}$ In strong fields $\left(H_{c 1} \ll H \ll H_{c 2}\right)$, each vortex overlaps with its neighborhood. Then the charge density outside the core is nearly constant and periodic modulation of the charge density appears for the periodic-vortex lattice [Fig. 1(b)]. In ordinary superconductors, $\left|Q_{\xi}\right|$ is estimated to be $\sim 10^{-5}-10^{-6} e$, using $k_{F} \sim 1 \AA^{-1}, \lambda_{T F} \sim k_{F}^{-1} \sim 1 \AA, \xi$ $\sim 100 \AA$, and $\left|d \ln T_{c} / d \ln \mu\right| \approx \ln \left(\hbar \omega_{D} / k_{B} T_{c}\right) \sim 1-10$, where 

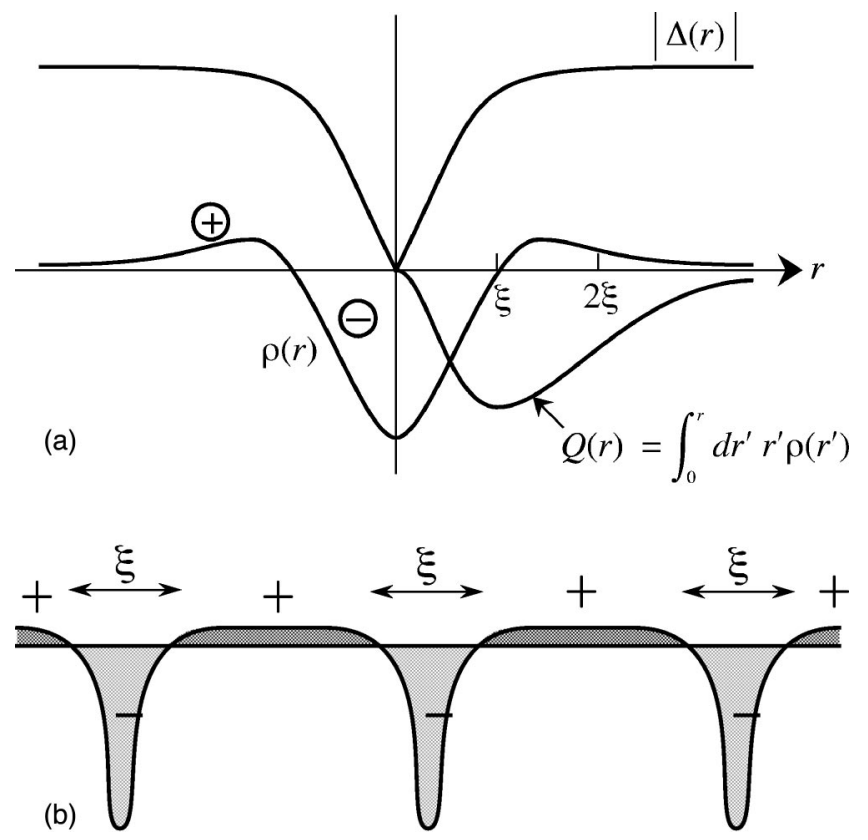

FIG. 1. (a) Schematic figure of the charge distribution around a single vortex core when the electrons are trapped within the core (negatively charged core). $\Delta(r)$ is the superconducting energy gap. $\rho(r)$ is the charge density. The charge accumulated inside the core is screened by the charges with opposite sign. $\rho(r)$ decays gradually as $r^{-4}$ well outside the core region. $Q(r)$ is the total charge within the distance $r$. $Q(r)$ goes to zero as $r \rightarrow \infty$ due to the requirement of the overall charge neutrality. (b) The charge-density modulation in the strong magnetic field $\left(H_{c 1} \ll H \ll H_{c 2}\right)$ where each vortices overlaps.

$\omega_{D}$ is the Debye frequency. Thus $\left|Q_{\xi}\right|$ is negligibly small and is very difficult to observe.

However, the situation in the case of HTSC seems to be promising because $\xi$ is extremely short compared to that of the conventional superconductors. Moreover, the strong electron-correlation effects and $d$-wave pairing symmetry of HTSC are expected to change the electronic structure of the vortex dramatically. ${ }^{12-17}$ In fact, recent scanning tunnel microscopy (STM) measurements revealed that the vortex of HTSC is very different from those of conventional superconductors. ${ }^{18-20}$ These unusual features of HTSC are expected to enhance the charging. We will discuss these issues later.

The vortex charge also plays an important role for the vortex dynamics. When a vortex moves within superfluid electrons, the core plays a key role in dissipation processes. ${ }^{10}$ One of the most striking phenomena is the vortex Hall anomaly, namely, sign reversal of the flux flow Hall effect below $T_{c}$, which is observed in most HTSC. ${ }^{21}$ This Hallsign reversal indicates that the vortices move upstream against the superfluid flow. Such an unusual motion has never been observed in any other fluid including superfluid helium and cannot be explained in the framework of the classical hydrodynamic theory. ${ }^{22}$ Recently, this phenomena has been discussed in terms of the vortex charge that produces an additional force acting on the vortices. ${ }^{6-9}$

To elucidate the vortex charge, a direct observation of the local carrier density is strongly required. It has been pointed out by many authors that $\nu_{Q}$ in HTSC is very sensitive to the local hole density. ${ }^{23-26}$ In a solid, an electron distribution with spherical asymmetry such as an unclosed $3 d$ shell and noncubic surrounding ions induce a local electric-field gradient (EFG) in the vicinity of the nuclei. This EFG lifts the degeneracy of the nuclear spin levels, interacting with the nuclear quadrupole moment $Q_{N}$. The relevant information is obtained from the nuclear quadrupole resonance (NQR) in zero magnetic field and the nuclear magnetic resonance (NMR) in a finite magnetic field. For the ${ }^{63} \mathrm{Cu}$ nuclei with spin $I=3 / 2$, the NQR resonance frequency $\nu_{Q}^{N Q R}$ is expressed as

$$
\nu_{Q}^{N Q R}=\frac{e^{2} Q_{N} q_{z z}}{2 h} \sqrt{1+\frac{\eta^{2}}{3}}=\nu_{Q} \sqrt{1+\frac{\eta^{2}}{3}}
$$

where $e q_{z z}$ is the largest principle ( $z$ axis) component of EFG at the nuclear site, $Q_{N}\left(=-0.211\right.$ barn for $\left.{ }^{63} \mathrm{Cu}\right)$ is the quadrupole moment of copper nuclei. ${ }^{27}$ The asymmetry parameter $\eta$ of the EFG defined as $\eta=\left|\left(q_{x x}-q_{y y}\right) / q_{z z}\right|$ is close to zero for the $\mathrm{Cu}$ site in the two-dimensional $\mathrm{CuO}_{2}$ planes $[\mathrm{Cu}(2)$ site $]$.

In a strong magnetic field, when the Zeeman energy is much larger than the quadrupole energy, each Zeeman level is shifted by the quadrupole interactions and thus, two satellite peaks $( \pm 3 / 2 \leftrightarrow \pm 1 / 2)$ appear on both sides of the central $( \pm 1 / 2 \leftrightarrow \mp 1 / 2)$ resonance peak. The frequency difference between the upper and the lower satellites exactly coincides with $2 \nu_{Q}$ when a magnetic field is applied parallel to the largest principle axis of EFG, namely, $H \|_{c}$ axis in the present experimental condition described below. Even for the second-order or more higher-order quadrupole effect in the presence of the asymmetry $\eta$ of EFG, each satellite line is shifted as much and the corrections for $2 \nu_{Q}$ are vanishing in the case of $H \| c$ axis. ${ }^{27}$

Generally the EFG originates from two different sources, namely, from the on-site distributions $q_{\text {on-site }}$ of the electrons and from the surrounding ions, $q_{i o n}, q=q_{o n-s i t e}+q_{i o n}$. Recent analysis of $q$ on the $\mathrm{Cu}(2)$ site suggests that $q_{\text {on-site }}$ is mainly composed of the $\mathrm{Cu} 4 p$ and $3 d$ shell terms. ${ }^{23}$ In HTSC the holes in the $\mathrm{Cu} 3 d_{x^{2}-y^{2}}$ orbital play an important role for the onset of superconductivity. Figure 2 shows the doping dependence of $\nu_{Q}$ of the $\mathrm{Cu}(2)$ site for $\mathrm{YBa}_{2} \mathrm{Cu}_{3} \mathrm{O}_{7-\delta},{ }^{24} \mathrm{La}_{2-x} \mathrm{Sr}_{x} \mathrm{CuO}_{4},{ }^{25}$ and $\mathrm{HaBa}_{2} \mathrm{CuO}_{4+\delta} \cdot{ }^{26}$ In all materials, $\nu_{Q}$ increases linearly with the number of holes in the planes and can be written as

$$
\nu_{Q}=A n_{\text {hole }}+C,
$$

where $n_{\text {hole }}$ is the number of holes per $\mathrm{Cu}(2)$ atom, and $A$ and $C$ are constants. ${ }^{24-26}$ Although $C$ is strongly material dependent, reflecting the difference in $\nu_{\text {ion }}, A \approx$ 20-30 MHz per hole for the $\mathrm{Cu}(2)$ atom is essentially material independent. Thus, the precise measurement of $\nu_{Q}$ makes possible an accurate determination of the change of the local hole number at the $\mathrm{Cu}$ site. 


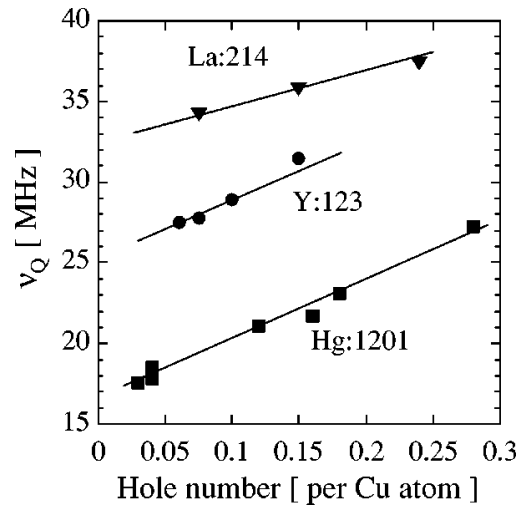

FIG. 2. Doping dependencies of $\nu_{Q}$ on the $\mathrm{Cu}(2)$ site for $\mathrm{YBa}_{2} \mathrm{Cu}_{3} \mathrm{O}_{7-\delta} \quad$ (Ref. 24), $\mathrm{La}_{2-x} \mathrm{Sr}_{x} \mathrm{CuO}_{4}$ (Ref. 25), and $\mathrm{HaBa}_{2} \mathrm{CuO}_{4+\delta}$ (Ref. 26). $\nu_{Q}$ is proportional to the hole number.

\section{EXPERIMENT}

The principle of our experiment is the following. In the measurement, only the resonance of the ${ }^{63} \mathrm{Cu}(2)$ nuclei outside the vortex core is detected. This is because the applied field is much less than $H_{c 2}$ and hence the core region occupies a smaller area in the sample. If the vortex core traps (expels) a finite amount of electrons, the electron density outside the core should decrease (increase) from that in zero field where the electron distribution is uniform, as shown in Fig. 1(b). We are able to detect the change of carrier density through the change of the value of $\nu_{Q}$.

In the present measurements, we used slightly overdoped $\mathrm{YBa}_{2} \mathrm{Cu}_{3} \mathrm{O}_{7}$ and underdoped $\mathrm{YBa}_{2} \mathrm{Cu}_{4} \mathrm{O}_{8}$ in which the NQR and NMR spectra are very sharp compared to those of other HTSC. The NMR spectra are obtained for fine-powdered samples (the grains are less than $33 \mu \mathrm{m}$ ) with uniaxial alignment. Each grain aligns to an easy axis (the $c$ axis) in a high external field at room temperature, and is then fixed with epoxy (Stycast 1266).

The Cu-NQR and NMR spectra were obtained by a conventional pulse spectrometer. The NMR experiments were performed in a field-cooling condition under a constant field of $9.4 \mathrm{~T}$ by using a highly homogeneous superconducting magnet that was stabilized to less than 1 ppm during the experiment. The present measurement in the vortex state is made in the so-called Bragg-glass phase in which the quasilong-range order of the vortex lattice is preserved. We see a slightly asymmetric shape of the NMR-satellite spectra due to the misalignment, somehow, of some grains. The line broadenings of the NQR spectra in zero external field are not observed even below $T_{c}$ in both $\mathrm{YBa}_{2} \mathrm{Cu}_{3} \mathrm{O}_{7}$ and $\mathrm{YBa}_{2} \mathrm{Cu}_{4} \mathrm{O}_{8}$. The width of the NMR spectra becomes broader with decreasing $T$ below $T_{c}$ partly due to the magnetic-field inhomogeneity caused by the introduction of the vortices and partly due to the spatial distribution of carrier density, which we will discuss later.

\section{RESULTS AND DISCUSSION}

Figures 3(a)-(c) show the NQR and NMR spectra of ${ }^{63} \mathrm{Cu}(2)$ for optimally doped $\mathrm{YBa}_{2} \mathrm{Cu}_{3} \mathrm{O}_{7}$. Figures $4(\mathrm{a})-(\mathrm{c})$
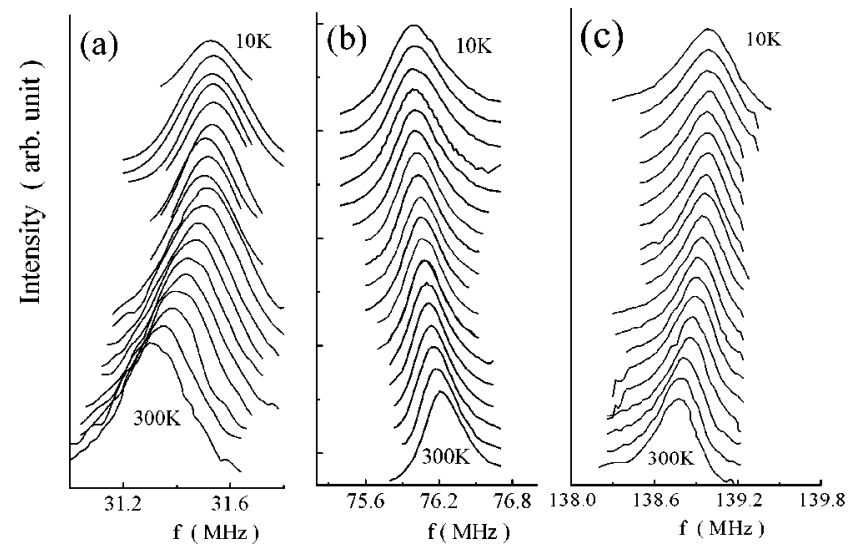

FIG. 3. The NQR (a) and the lower (b), and upper (c) NMR satellite spectra at $9.4 \mathrm{~T}$ for ${ }^{63} \mathrm{Cu}(2)$ site of $\mathrm{YBa}_{2} \mathrm{Cu}_{3} \mathrm{O}_{7}$ for various temperatures $(220 \mathrm{~K}, 200 \mathrm{~K}, 180 \mathrm{~K}$, and $160-10 \mathrm{~K}$ in a $10-\mathrm{K}$ step).

show the same spectra for $\mathrm{YBa}_{2} \mathrm{Cu}_{4} \mathrm{O}_{8}$. All data were taken by utilizing a phase-coherent spin-echo spectrometer. The spectra are obtained with a superposed method of the Fourier-transform spectra of the spin echo measured at a certain frequency interval. The temperature dependencies of $\nu_{Q}$ obtained from NMR and NQR are shown for $\mathrm{YBa}_{2} \mathrm{Cu}_{3} \mathrm{O}_{7}$ and $\mathrm{YBa}_{2} \mathrm{Cu}_{4} \mathrm{O}_{8}$ in Figs. 5 and 6, respectively. The temperature dependencies of $\nu_{Q}^{N Q R}$ for both $\mathrm{YBa}_{2} \mathrm{Cu}_{3} \mathrm{O}_{7}$ and $\mathrm{YBa}_{2} \mathrm{Cu}_{4} \mathrm{O}_{8}$ are quite similar to those of $\nu_{Q}^{N Q R}$ reported previously. ${ }^{28}$ It should be noted that the procedure for obtaining $\nu_{Q}$ from the frequency difference between the upper and lower satellites is essentially free from the influence of the change of the magnetic shift (or Knight shift). Moreover, the magnetic effect of the asymmetric broadening ${ }^{29}$ (so-called Redfield pattern) due to the vortex lattice in the superconducting state is exactly canceled out in the process determining $\nu_{Q}$ from NMR. Thus, we obtained the $\nu_{Q}$ values simply from the difference of the peak frequencies of the two satellite lines.
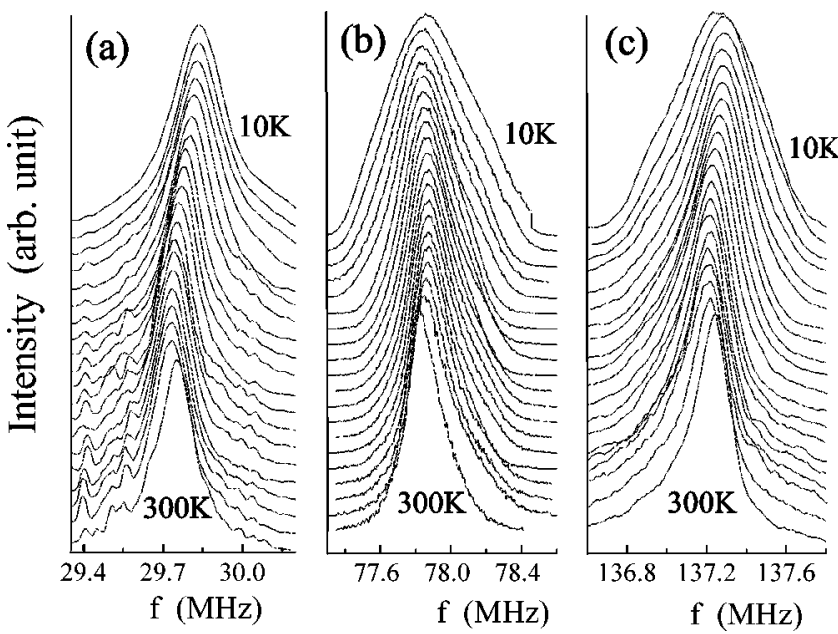

FIG. 4. The NQR in zero field (a) and the lower (b) and upper (c) NMR satellite spectra at $9.4 \mathrm{~T}$ for ${ }^{63} \mathrm{Cu}(2)$ site of $\mathrm{YBa}_{2} \mathrm{Cu}_{4} \mathrm{O}_{8}$ for various temperatures $(300 \mathrm{~K}, 220 \mathrm{~K}, 200 \mathrm{~K}, 180 \mathrm{~K}$, and $160-10$ $\mathrm{K}$ in a $10-\mathrm{K}$ step). 


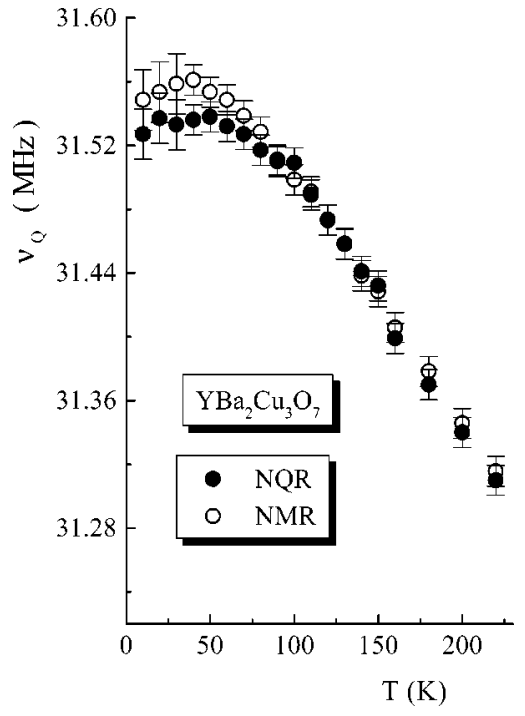

FIG. 5. The temperature dependence of $\nu_{Q}$ obtained from NQR and NMR for ${ }^{63} \mathrm{Cu}(2)$ site of $\mathrm{YBa}_{2} \mathrm{Cu}_{3} \mathrm{O}_{7}$.

We plot in Fig. 7 the difference between $\nu_{Q}$ in zero field and in the vortex state, $\Delta \nu_{Q}=\nu_{Q}(0)-\nu_{Q}(H)$, for $\mathrm{YBa}_{2} \mathrm{Cu}_{3} \mathrm{O}_{7}$ and $\mathrm{YBa}_{2} \mathrm{Cu}_{4} \mathrm{O}_{8}$. The $\nu_{Q}$ in zero field for $\mathrm{NQR}$ is obtained after correction by the factor of $\sqrt{1+\eta^{2} / 3}$ in Eq. (1), although this factor is at most $0.03 \%$ of $\nu_{Q}$ for $\eta$ $\approx 0.04$ of the present materials. In both materials $\Delta \nu_{Q}$ is essentially zero above $T_{c}$, indicating no modulation of the carrier density. Meanwhile a nonvanishing $\Delta \nu_{Q}$ is clearly observed below $T_{c}$ in both materials. While $\Delta \nu_{Q} \sim$ $-25 \mathrm{kHz}$ is negative in $\mathrm{YBa}_{2} \mathrm{Cu}_{3} \mathrm{O}_{7}, \Delta \nu_{Q} \sim 50 \mathrm{kHz}$ is positive in $\mathrm{YBa}_{2} \mathrm{Cu}_{4} \mathrm{O}_{8}$ at $T=0$.

We discuss here several possible origins for the nonzero $\Delta \nu_{Q}$. We first point out that the magnetostriction cannot be the origin of the nonzero $\Delta \nu_{Q}$. In fact, both the magnetostriction and ultrasonic-absorption measurements showed that the local lattice distortion $\Delta l$ caused by the magnetostriction is negligibly small under the field-cooling condition; $\Delta l / l<10^{-8}$ below $10 \mathrm{~T}$ where $l$ is the lattice constant. We

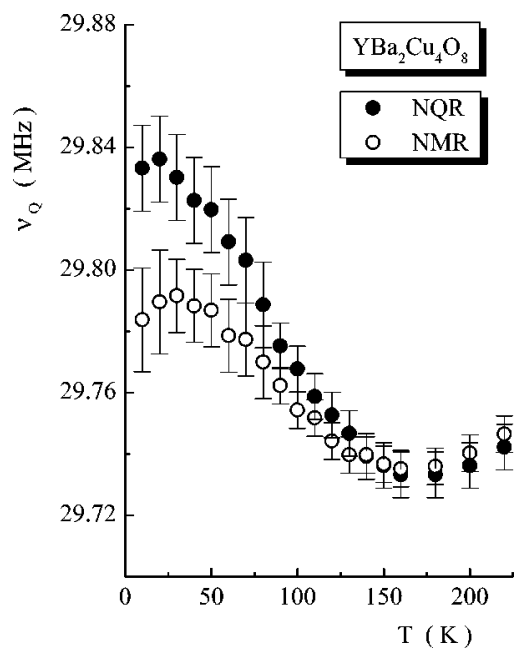

FIG. 6. The temperature dependence of $\nu_{Q}$ obtained from NQR and NMR for ${ }^{63} \mathrm{Cu}(2)$ site of $\mathrm{YBa}_{2} \mathrm{Cu}_{4} \mathrm{O}_{8}$.

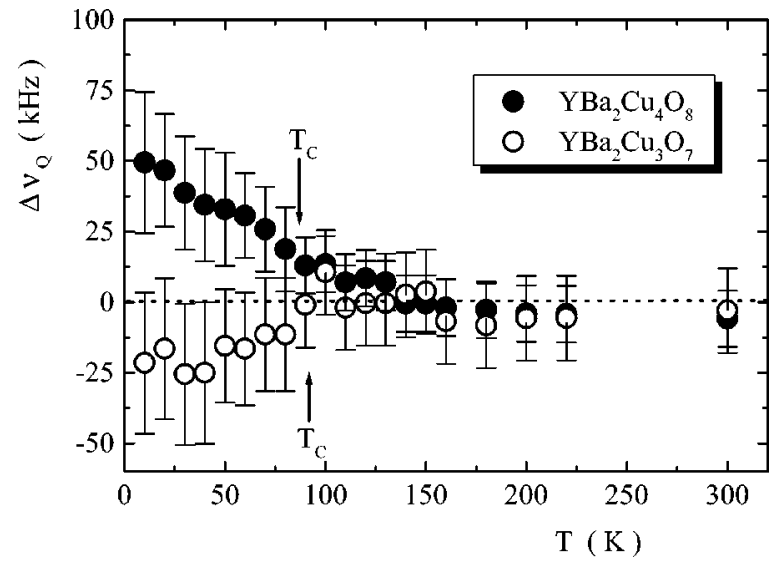

FIG. 7. $T$ dependence of $\Delta \nu_{Q}=\nu_{Q}(0)-\nu_{Q}(H)$ for $\mathrm{YBa}_{2} \mathrm{Cu}_{3} \mathrm{O}_{7}$ and $\mathrm{YBa}_{2} \mathrm{Cu}_{4} \mathrm{O}_{8}$. In both materials nonzero $\Delta \nu_{Q}$ is clearly observed below $T_{c}$, showing that the electron density outside the core differs from that in zero field.

next remark on an in-plane charge modulation caused by a charge-stripe formation ${ }^{30}$ or the charge density wave (CDW) transition of the chain site. ${ }^{31,32}$ Although the static-charge ordering associated with a stripe formation has been discussed in some of the high- $T_{c}$ cuprates, such orderings have never been reported in $\mathrm{YBa}_{2} \mathrm{Cu}_{3} \mathrm{O}_{7}$ nor in $\mathrm{YBa}_{2} \mathrm{Cu}_{4} \mathrm{O}_{8}$. Moreover, it has been reported that in La:214 compounds the static-charge-stripe order gives rise to the wipeout of NQR signals, ${ }^{33}$ implying that the static-stripe order, if present, induces large effects on quadrupole interactions. However, we have observed neither the broadening of the NQR spectra (in zero field) nor any sign of the wipeout of NQR and NMR signals in the present measurements for $\mathrm{YBa}_{2} \mathrm{Cu}_{3} \mathrm{O}_{7}$ or $\mathrm{YBa}_{2} \mathrm{Cu}_{4} \mathrm{O}_{8}$. Finally we mention a recent report of the broadening of the NQR line widths in the plane at low temperatures in $\mathrm{YBa}_{2} \mathrm{Cu}_{3} \mathrm{O}_{7-\delta}$, which was explained in terms of the CDW formation in the chain site. ${ }^{32}$ In the present study, in which we obtained sharper NQR line width compared to the previous reports, ${ }^{31,32}$ such line broadenings due to the electric-quadrupole interaction is not observed. Thus, there is no evidence of charge modulation due to CDW or stripe formation in zero field in our crystals. It is also quite unlikely that the magnetic field induces the CDW or stripe formations. Having ruled out these various possibilities, we conclude that the nonzero values for $\Delta \nu_{Q}$ naturally lead to the fact that the electron density outside the vortex core is different from that in zero field.

We now discuss the issue of the sign and magnitude of the accumulated charge. The negative $\Delta \nu_{Q}$ in $\mathrm{YBa}_{2} \mathrm{Cu}_{3} \mathrm{O}_{7}$ indicates the increment of the hole density outside the core. This excess density of holes is nothing but the holes expelled from the core. Therefore, the accumulated charge in the core of $\mathrm{YBa}_{2} \mathrm{Cu}_{3} \mathrm{O}_{7}$ is negative. By the same reasoning, the positive $\Delta \nu_{Q}$ in $\mathrm{YBa}_{2} \mathrm{Cu}_{4} \mathrm{O}_{8}$ indicates a positive accumulated charge. Meanwhile, since the chemical potential decreases monotonically with doping holes, Eq. (1), $\operatorname{sgn} Q_{\xi}$ $=\operatorname{sgn}\left(d \ln T_{c} / d \ln \mu\right)$, predicts that $Q_{\xi}>0$ in the underdoped regime while $Q_{\xi}<0$ in the overdoped regime. This is strikingly in contrast to the sign determined by the present experiment. The deviation of the magnitude of the charge from 
theory is also noteworthy. The magnitude of charges per pancake vortex, which are roughly estimated using $Q_{\xi}$ $\approx \Delta \nu_{Q} H_{c 2} / A H$ assuming $H_{c 2} \sim 200 \mathrm{~T}$ are $Q_{\xi} \sim-0.005 e$ to $-0.02 e$ for $\mathrm{YBa}_{2} \mathrm{Cu}_{3} \mathrm{O}_{7}$ and $Q_{\xi} \sim 0.01 e-0.05 e$ for $\mathrm{YBa}_{2} \mathrm{Cu}_{4} \mathrm{O}_{8}$. However, according to Eq. (1), $Q_{\xi}$ is estimated to be $\sim 10^{-4}-10^{-5} e$ where we assumed $\xi \sim 30 \AA$. Therefore, $\left|Q_{\xi}\right|$ determined by the present experiments are still one or two orders of magnitude larger than expected by Eq. (1). Thus the BCS theory not only predicts the wrong sign of the charge but also underestimates $\left|Q_{\xi}\right|$ greatly.

There are several intriguing possible origins for these discrepancies. For example, because of the extremely short $\xi$, the vortex core may be in the quantum limit $k_{F} \xi \sim 1$, where $k_{F}$ is the Fermi wave number. In this limit, the description of the quasiparticles in terms of semiclassical wave packets breaks down in contrast to conventional superconductors. ${ }^{13}$ Furthermore, as suggested by recent theories of the vortex core based on, e.g., the $t-J$ or $\mathrm{SO}(5)$ models, the antiferromagnetic (AF) state may be energetically preferable to the metallic state in the vortex core of HTSC. ${ }^{14,15,17}$ If this is indeed so, the AF correlation is expected to enhance the charging effect because it causes a large shift in $\mu$ by changing the density of states of the electrons inside the core dramatically. We note here that the present results exclude the possibility of the $\mathrm{SO}(5)$-insulating $\mathrm{AF}$ core $^{14,17}$ in which holes should be expelled from the core and the accumulated charges are always negative; the present result yields the opposite sign for $\mathrm{YBa}_{2} \mathrm{Cu}_{4} \mathrm{O}_{8}$ in the underdoped regime where the AF correlation is important. Therefore, a detailed microscopic calculation is needed to evaluate the accumulated charge quantitatively including the sign.

\section{CONCLUSION}

From the precise ${ }^{63} \mathrm{Cu}-\mathrm{NMR}$ and NQR measurements, we have shown that a vortex in type-II superconductors can trap a finite electric charge as well as magnetic flux. In the slightly overdoped $\mathrm{YBa}_{2} \mathrm{Cu}_{3} \mathrm{O}_{7}$ the vortex is negatively charged, while in underdoped $\mathrm{YBa}_{2} \mathrm{Cu}_{4} \mathrm{O}_{8}$ it is positively charged. In both high $T_{c}$ materials, the accumulated charges are much larger than expected in the ordinary superconductors. The sign and value of the charges indicate the electronic structure of the vortex in HTSC.

\section{ACKNOWLEDGMENTS}

We thank Y. Kato, K. Maki, T. Mishonov, M. Ogata, D. Rainer, E. B. Sonin, M. Takigawa, A. Tanaka, and H. Yasuoka for helpful discussions. We also thank H. Ikuta and T. Hanaguri for comments on the magnetostriction. We also thank S. Shamoto and T. Isobe for their technical assistance on the crystal growth and K. Kakuyanagi for his assistance on the NMR measurement. This work was supported by the REIMEI Research Resources of the Japan Atomic Energy Research Institute, Iketani Science and Technology Foundation and a Grant-in-Aid for Scientific Research on Priority Area "Vortex Electronics" from the Ministry of Education, Science, Sports and Culture of Japan.
${ }^{1}$ P. G. de Gennes, Superconductivity of Metals and Alloys (Addison-Wesley, Reading, MA, 1989).

${ }^{2}$ D. I. Khomskii and A. Freimuth, Phys. Rev. Lett. 75, 1384 (1995).

${ }^{3}$ G. Blatter et al., Phys. Rev. Lett. 77, 566 (1996).

${ }^{4}$ T. Mishonov, cond-mat/0004286 (unpublished).

${ }^{5}$ H. Hayashi et al., J. Phys. Soc. Jpn. 67, 3368 (1999); J. Kolacek et al., Phys. Rev. Lett. 86, 312 (2001).

${ }^{6}$ A. T. Dorsey et al., Phys. Rev. B 46, 8376 (1992); N. B. Kopnin and A. V. Lopatin, ibid. 51, 15291 (1995).

${ }^{7}$ A. van Otterlo et al., Phys. Rev. Lett. 75, 3736 (1995).

${ }^{8}$ M. V. Feigel'man et al., Pis'ma Zh. Éksp. Teor. Fiz. 62, 811 (1995) [JETP Lett. 62, 834 (1995)].

${ }^{9}$ Y. Kato, J. Phys. Soc. Jpn. 68, 3798 (1999).

${ }^{10}$ M. Eschrig et al., Phys. Rev. B 60, 10447 (1999).

${ }^{11}$ F. Gygi and M. Schlüter, Phys. Rev. B 43, 7609 (1991).

${ }^{12}$ N. Schopohl and K. Maki, Phys. Rev. B 52, 490 (1995).

${ }^{13}$ Y. Morita et al., Phys. Rev. Lett. 78, 4841 (1997).

${ }^{14}$ D. P. Arovas et al., Phys. Rev. Lett. 79, 2871 (1997).

${ }^{15}$ A. Himeda et al., J. Phys. Soc. Jpn. 66, 3367 (1997); H. Han and D. H. Lee, Phys. Rev. Lett. 85, 1100 (2000).

${ }^{16}$ M. Franz and Z. Tesanovic, Phys. Rev. Lett. 80, 4763 (1998).

${ }^{17}$ B. M. Andersen et al., Phys. Rev. B 61, 6298 (2000).

${ }^{18}$ I. Maggio-Aprile et al., Phys. Rev. Lett. 75, 2754 (1995).
${ }^{19}$ Ch. Renner et al., Phys. Rev. Lett. 80, 3606 (1998).

${ }^{20}$ S. H. Pan et al., Phys. Rev. Lett. 85, 1536 (2000).

${ }^{21}$ T. Nagaoka et al., Phys. Rev. Lett. 80, 3594 (1998), and references therein.

${ }^{22}$ J. Bardeen and M. J. Stephen, Phys. Rev. 140, A1197 (1965); P. Noziéres and W. F. Vinen, Philos. Mag. 14, 667 (1966).

${ }^{23}$ K. Schwarz, Phys. Rev. B 42, 2051 (1990); Y. Ohta et al., J. Phys. Soc. Jpn. 61, 2198 (1992).

${ }^{24} \mathrm{H}$. Yasuoka, in Spectroscopy of Mott Insulator and Correlated Metals, edited by A. Fujimori and Y. Tokura, Solid State Sciences Vol. 119 (Springer-Verlag, Berlin, 1995), p. 213.

${ }^{25}$ G. Zheng et al., J. Phys. Soc. Jpn. 64, 2524 (1995).

${ }^{26}$ A. A. Gippius et al., Physica C 276, 57 (1997).

${ }^{27}$ G. C. Cater et al., in Metallic Shifts in NMR (Pergamon Press, New York, 1977).

${ }^{28}$ K. Muller et al., Physica C 162-164, 173 (1989); A. Suter et al., Phys. Rev. B 56, 5542 (1997).

${ }^{29}$ H. Ikuta et al., Phys. Rev. Lett. 70, 2166 (1993); T. Hagaguri (private communication).

${ }^{30}$ J. M. Tranquada et al., Nature (London) 375, 561 (1995).

${ }^{31}$ S. Kramer and M. Mehing, Phys. Rev. Lett. 83, 396 (1999); 84, 1637 (2000).

${ }^{32}$ B. Grevin, Y. Berthier, and G. Collin, Phys. Rev. Lett. 85, 1310 (2000); 84, 1636 (2000).

${ }^{33}$ A. W. Hunt et al., Phys. Rev. Lett. 82, 4300 (1999). 